\title{
UDC 621.793.927.7
}

\section{OPTIMAL CONTROL OF INDUCTION HEATING IN THE SURFACING PROCESSES OF THIN SHAPED DISKS}

\author{
Volodymyr Havryliuk; Volodymyr Mykhailyshyn; \\ Mykhailo Mykhailyshyn; Cheslav Pulka
}

\author{
Ternopil Ivan Puluj National Technical University, Ternopil, Ukraine
}

\begin{abstract}
Summary. Induction heating is used by technological processes of surfacing. The advantages of induction heating are high concentration of energy at the heating area that allows to increase the productivity of process, because time of surfacing decreases. The disadvantages of induction heating are electromagnetic radiation and excessive loss of electrical energy. The authors deal with development of the optimal law for source capacity change by using methods of calculation. It allows to reduce electrical energy consumption by 36,8\% and 15,8\% compared with traditional laws of capacity distribution.
\end{abstract}

Key words: induction heating, surfacing, optimal law, capacity, temperature, thin shaped disks.

Statement of the problem. For disk outer edge surfacing with wear-resistant material an induction coil is used [1-5], which allows to perform simultaneous surfacing onto the entire area being reinforced. This type of surfacing ensures axial symmetry of the process, thus we obtain the part without any distortions and no further trimming is needed. Surfacing is performed by using induction heating, its advantages being high energy concentration within the heating area, which allows to increase productivity of the process by reducing time $[6,7]$. Still, the induction heating has a number of disadvantages, - namely the electromagnetic radiation and excessive electrical energy consumption, which in their turn had negative impact on people involved and high rate of energy consumption.

Evaluation of known experimental studies. Work [8] provides approximate formula for time-dependent capacity of heat sources to calculate the amount of required temperature to be applied onto surfacing area within specified time $\tau^{*}$ at lowest consumption of energy.

Objective of the research. The objective of this study is to produce more accurate optimal time law for capacity of heat sources, which would allow to considerably reduce energy consumption hence saving funds and resources.

Statement of the task. As energy conservation is of great importance for high-quality surfacing, a demand arises for finding out more accurate optimal time law for capacity of heat sources.

Certain temperature $T_{z d}^{*}$ should be provided in the thin shaped disc with radius $R_{2}$ in the area of outer edge $R_{1}<r<R_{2}$ to ensure high-quality surfacing at lowest consumption of energy.

The equation of disc thermal conductivity for averaged in thickness temperature and averaged capacity of heat sources is $[8,9]$ 


$$
\frac{\partial^{2} T}{\partial r^{2}}+\frac{1}{r} \frac{\partial T}{\partial r}-\frac{k^{*}}{h} T-\frac{1}{a} \frac{\partial T}{\partial t}+\frac{w(r, t)}{\lambda}=0,
$$

where $T=T^{*}-T_{\mathrm{cp}}, T^{*}$ is disc temperature, $T_{\mathrm{cp}}=$ const - ambient temperature; $k^{*}=\alpha \lambda$; $\alpha$ - heat-transfer coefficient; $\lambda$ - thermal conductivity of the disc material; $2 h-$ thickness of the disc; $a$ - thermal diffusivity; $w(r, t)$ - averaged in thickness function of specific capacity of inner heat sources.

It is considered that if $r=R_{2}$, convective heat transfer condition comes in place and there is no heat flow through the center of the disc, i.e. the following boundary conditions occur:

$$
\begin{gathered}
\frac{\partial T}{\partial r}=0 \text { if } r=0, \\
\frac{\partial T}{\partial r}+k^{*} T=0 \text { if } r=R_{2} .
\end{gathered}
$$

At zero moment the disc temperature is constant and equal to ambient temperature. So the initial condition is the following:

$$
T=0 \text { if } t=0 \text {. }
$$

It is necessary to produce the law of heat sources capacity change at lowest energy consumption needed for surfacing, provided the final temperature distribution within the disc has the least predetermined deviation value. Surfacing time is considered to be predetermined and equal to $\tau^{*}$.

Thus, it is necessary to minimize the composite function

$$
I=\int_{0}^{R_{2}}\left[T\left(r, \tau^{*}\right)-T_{z d}(r)\right]^{2} r d r+\int_{0}^{\tau^{*}} \int_{0}^{R_{2}}\left(\frac{a w(r, t)}{\lambda}\right)^{2} r d r d t \Rightarrow \min
$$

provided, the process is described by equation (1) under boundary and initial conditions (2-4).

Extended composite function of the task is as follows

$$
\begin{aligned}
& J=\int_{0}^{R_{2}}\left[T\left(r, \tau^{*}\right)-T_{z d}(r)\right]^{2} r d r+\int_{0}^{\tau^{*}} \int_{0}^{R_{2}}\left(\frac{a w(r, t)}{\lambda}\right)^{2} r d r d t+ \\
& +\int_{0}^{\tau^{*}} \int_{0}^{R_{2}} \bar{T}(r, t)\left(\frac{\partial^{2} T}{\partial r^{2}}+\frac{1}{r} \frac{\partial T}{\partial r}-\frac{k^{*}}{h} T-\frac{1}{a} \frac{\partial T}{\partial t}+\frac{w(r, t)}{\lambda}\right) r d r d t,
\end{aligned}
$$

where $\bar{T}(r, t)$ is Lagrangian multiplier. variation

Necessary criterion for composite function minimum is zero equality of the first 


$$
\delta J=0
$$

Having found the first variation and equaled it to zero, the conjugate problem is produced:

$$
\begin{gathered}
\frac{\partial^{2} \bar{T}}{\partial r^{2}}+\frac{1}{r} \frac{\partial \bar{T}}{\partial r}-\frac{k^{*}}{h} \bar{T}+\frac{1}{a} \frac{\partial \bar{T}}{\partial t}=0, \\
\frac{\partial \bar{T}}{\partial r}+k^{*} \bar{T}=0 \text { if } r=R_{2}, \\
\bar{T}\left(r, \tau^{*}\right)=2 a\left[T\left(r, \tau^{*}\right)-T_{z d}(r)\right],
\end{gathered}
$$

as well as the following formula for optimal distribution of inner sources:

$$
w(r, t)=-\frac{\lambda}{2 a^{2}} \bar{T}(r, t)
$$

Apart from this, a direct problem (1)-(4) should be performed. The solution of the conjugant problem, produced by Fourier method, is

$$
\bar{T}(r, t)=\sum_{k=1}^{\infty} A_{k} e^{a \omega_{\mathrm{K}}^{2} t} J_{0}\left(\nu_{\mathrm{K}} r\right)
$$

where $J_{0}\left(v_{\mathrm{K}} r\right)$ - zero-order Bessel function, $v_{k}$ - roots of the characteristic equation.

$$
\begin{gathered}
k^{*} J_{0}\left(v R_{2}\right)-v J_{1}\left(v R_{2}\right)=0 . \\
\omega_{\mathrm{K}}^{2}=v_{\mathrm{K}}^{2}+\frac{k^{*}}{h} .
\end{gathered}
$$

Solution (12) does not yet fulfils condition (10). To satisfy this condition we should find the solution of the direct problem. Taking into account (11) and (12) the equation (1) will be the following:

$$
\frac{\partial^{2} T}{\partial r^{2}}+\frac{1}{r} \frac{\partial T}{\partial r}-\frac{k^{*}}{h} T-\frac{1}{a} \frac{\partial T}{\partial t}=\frac{1}{2 a^{2}} \sum_{k=1}^{\infty} A_{k} e^{a \omega_{\mathrm{K}}^{2} t} J_{0}\left(v_{\mathrm{K}} r\right)
$$

As fundamental functions in the coordinate $r$ and eigenvalues are the same as for conjugant problem, we are seeking the solution of the problem (15) in the form of: 


$$
T(r, t)=\sum_{k=1}^{\infty} \theta_{\mathrm{K}}(t) J_{0}\left(v_{\mathrm{K}} r\right)
$$

Functions $\theta_{\mathrm{K}}(t)$, based on the initial condition (4) should fulfil condition

$$
\theta_{\mathrm{K}}(0)=0 \text {. }
$$

Having substituted (16) and (15), the following equation for finding functions $\theta_{\mathrm{K}}(t)$ is produced

$$
\frac{d \theta_{\mathrm{K}}}{d t}+a \omega_{\mathrm{K}}^{2} \theta_{\mathrm{K}}=-\frac{A_{\mathrm{K}}}{2 a} e^{a \omega_{\mathrm{K}}^{2} t}
$$

The general solution, if condition (17) is taken into account, can be the following:

$$
\theta_{\mathrm{\kappa}}(t)=-\frac{A_{\mathrm{K}}}{2 a^{2} \omega_{\mathrm{K}}^{2}} \operatorname{sh}\left(a \omega_{\mathrm{\kappa}}^{2} t\right)
$$

So, the solution of the direct problem is

$$
T(r, t)=-\sum_{\mathrm{K}=1}^{\infty} \frac{1}{2 a^{2} \omega_{\mathrm{K}}^{2}} A_{\mathrm{K}} \operatorname{sh}\left(a \omega_{\mathrm{K}}^{2} t\right) J_{0}\left(v_{\mathrm{K}} r\right)
$$

To satisfy condition (10) function $T_{z d}(r)$ is expanded in fundamental functions of the problem

$$
\begin{gathered}
T_{z d}(r)=\sum_{\mathrm{\kappa}=1}^{\infty} b_{\mathrm{K}} J_{0}\left(v_{\mathrm{K}} r\right) . \\
b_{\mathrm{K}}=\frac{2}{R_{2}^{2} J_{1}^{2}\left(v_{\mathrm{K}} R_{2}\right)} \int_{0}^{R_{2}} T_{z d}(r) J_{0}\left(v_{\mathrm{K}} r\right) r d r
\end{gathered}
$$

Having fulfilled (10), we obtain

$$
A_{\mathrm{K}}=-\frac{2 a^{2} \omega_{\mathrm{K}}^{2} \varphi_{\mathrm{K}}}{a \omega_{\mathrm{K}}^{2} e^{2 a \omega_{\mathrm{K}}^{2} \tau^{*}}+\operatorname{sh}\left(\omega_{\mathrm{K}}^{2} \tau^{*}\right)} .
$$

Thus, the optimal law for distribution of source capacity is 


$$
w(r, t)=\lambda \cdot \sum_{\mathrm{K}=1}^{\infty} \frac{\omega_{\mathrm{K}}^{2} b_{\mathrm{K}} e^{a \omega_{\mathrm{K}}^{2} t}}{a \omega_{\mathrm{K}}^{2} e^{a \omega_{\mathrm{K}}^{2} \tau^{*}}+\operatorname{sh}\left(a \omega_{\mathrm{K}}^{2} \tau^{*}\right)} \cdot J_{0}\left(v_{\mathrm{K}} r\right) .
$$
follows:

The corresponding function of average temperature distribution within the disc is as

$$
T(r, t)=\sum_{\mathrm{\kappa}=1}^{\infty} \frac{b_{\mathrm{K}} \operatorname{sh}\left(a \omega_{\mathrm{K}}^{2} t\right)}{a \omega_{\mathrm{K}}^{2} e^{a \omega_{\mathrm{K}}^{2} \tau^{*}}+\operatorname{sh}\left(a \omega_{\mathrm{K}}^{2} \tau^{*}\right)} \cdot J_{0}\left(v_{\mathrm{K}} r\right) .
$$

As a result of induction heat the predetermined temperature $T_{z d}^{*}$ should be reached within the area of surfacing $R_{1} \leq r \leq R_{2}$, we define

$$
T_{z d}(r)=\left\{\begin{aligned}
0, & 0 \leq r \leq R_{1} \\
T_{z d}^{*}, & R_{1} \leq r \leq R_{2}
\end{aligned}\right.
$$

In this case the distribution coefficient (22) will be equal

$$
b_{\mathrm{K}}=\frac{2 k^{* 2} T_{z d}^{*}\left[R_{2} J_{1}\left(v_{\mathrm{K}} R_{2}\right)-R_{1} J_{1}\left(v_{\mathrm{K}} R_{1}\right)\right]}{R_{2}^{2} \cdot v_{\mathrm{K}}\left(v_{k}^{2}+k^{* 2}\right) \cdot J_{1}^{2}\left(v_{\mathrm{K}} R_{2}\right)} .
$$

As a result, distribution functions of optimal heat source capacity and temperature field will be as follows:

$$
\begin{gathered}
w(r, t)=\frac{2 \lambda k^{* 2} T_{z d}^{*}}{R_{2}^{2}} \sum_{\mathrm{K}=1}^{\infty} \frac{\omega_{\mathrm{K}}^{2}\left[R_{2} J_{1}\left(v_{\mathrm{K}} R_{2}\right)-R_{1} J_{1}\left(v_{\mathrm{K}} R_{1}\right)\right] e^{a \omega_{\mathrm{K}}^{2} t} J_{0}\left(v_{\mathrm{K}} r\right)}{\left.v_{k}^{2}+{k^{*}}^{2}\right) J_{1}^{2}\left(v_{\mathrm{K}} R_{2}\right)\left(a \omega_{\mathrm{K}}^{2} e^{a \omega_{\mathrm{K}}^{2} \tau^{*}}+\operatorname{sh}\left(a \omega_{\mathrm{K}}^{2} \tau^{*}\right)\right)}, \\
T(r, t)=\frac{2 k^{* 2} T_{z d}^{*}}{R_{2}^{2}} \sum_{\mathrm{K}=1}^{\infty} \frac{\left[R_{2} J_{1}\left(v_{\mathrm{K}} R_{2}\right)-R_{1} J_{1}\left(v_{\mathrm{K}} R_{1}\right)\right] \operatorname{sh}\left(a \omega_{\mathrm{K}}^{2} t\right) J_{0}\left(v_{\mathrm{K}} r\right)}{v_{\mathrm{K}}\left(v_{k}^{2}+k^{* 2}\right) J_{1}^{2}\left(v_{\mathrm{K}} R_{2}\right)\left(a \omega_{\mathrm{K}}^{2} e^{a \omega_{\mathrm{K}}^{2} \tau^{*}}+\operatorname{sh}\left(a \omega_{\mathrm{K}}^{2} \tau^{*}\right)\right)} .
\end{gathered}
$$

Heat sources will be concentrated within the surfacing area under induction coil. Therefore we average the source strength by the surfacing area width

$$
\begin{gathered}
w_{0}(t)=\frac{2}{R_{2}^{2}-R_{1}^{2}} \int_{R_{1}}^{R_{2}} w(r, t) r d r= \\
=\frac{4 \lambda k^{* 2} T_{z d}^{*}}{R_{2}^{2}\left(R_{2}^{2}-R_{1}^{2}\right)} \sum_{\mathrm{K}=1}^{\infty} \frac{\omega_{\mathrm{K}}^{2} e^{a \omega_{\mathrm{K}}^{2} t}\left[R_{2} J_{1}\left(v_{\mathrm{K}} R_{2}\right)-R_{1} J_{1}\left(v_{\mathrm{K}} R_{1}\right)\right]^{2}}{v_{k}^{2}\left(v_{k}^{2}+k^{* 2}\right) J_{1}^{2}\left(v_{\mathrm{K}} R_{2}\right)\left(a \omega_{\mathrm{K}}^{2} e^{a \omega_{\mathrm{K}}^{2} \tau^{*}}+\operatorname{sh}\left(a \omega_{\mathrm{K}}^{2} \tau^{*}\right)\right)} .
\end{gathered}
$$

Full specific energy during the entire time of heating $\tau^{*}$ equals 


$$
\begin{gathered}
W_{\text {opt }}=\int_{0}^{\tau^{*}} w_{0}(t) d t= \\
=\frac{4 \lambda k^{* 2} T_{z d}^{*}}{a R_{2}^{2}\left(R_{2}^{2}-R_{1}^{2}\right)} \sum_{\mathrm{K}=1}^{\infty} \frac{\left(e^{a \omega_{\mathrm{K}}^{2} \tau^{*}}-1\right)\left[R_{2} J_{1}\left(v_{\mathrm{K}} R_{2}\right)-R_{1} J_{1}\left(v_{\mathrm{K}} R_{1}\right)\right]^{2}}{v_{k}^{2}\left(v_{k}^{2}+k^{* 2}\right) J_{1}^{2}\left(v_{\mathrm{K}} R_{2}\right)\left(a \omega_{\mathrm{K}}^{2} e^{a \omega_{\mathrm{K}}^{2} \tau^{*}}+\operatorname{sh}\left(a \omega_{\mathrm{K}}^{2} \tau^{*}\right)\right)} .
\end{gathered}
$$

In order to evaluate energy conservation, which is occurs as a result of specific capacity variation of sources according to the optimal law (28), we need to find the value of constant in time specific capacity of sources which is needed to heat the surfacing area of the disc to the predetermined average temperature $T_{z d}^{*}$. It can be shown [8] that this value equals

$$
w^{*}=\frac{\lambda k^{*} T_{z d}^{*}}{h\left(1-e^{-\frac{a k^{*} \tau^{*}}{h}}\right)}
$$

and correspondingly full specific energy during the entire time of heating

$$
W^{*}=\frac{\lambda k^{*} T_{z d}^{*} \tau^{*}}{h\left(1-e^{-\frac{a k^{*} \tau^{*}}{h}}\right)}
$$

Energy conservation, which is obtained due to heating according to optimal law, equals

$$
\Delta_{1}=\frac{W_{o p t}-W^{*}}{W^{*}} \cdot 100 \%
$$

It also is interesting to compare full energy consumption needed for heating, which was found in this research, with equivalent value, provided the change of capacity of inner sources is performed according to optimal law found in work [8] and which equals

$$
W_{s h}=\frac{\lambda T_{z d}^{*}}{a * \operatorname{sh}\left(\frac{a k^{*} \tau^{*}}{h}\right)} \cdot\left(e^{\frac{a k^{*} \tau^{*}}{h}}-1\right)
$$

Correspondingly the energy conservation equals

$$
\Delta_{2}=\frac{W_{o p t}-W_{s h}}{W_{s h}} \cdot 100 \%
$$

Numerical calculations are performed for the following data. Geometrical dimensions $-R_{1}=0,092 m, R_{2}=0,105 m, 2 h=0,003 m$. Disc material - steel St3sp with thermal and physical properties: thermal diffusivity- $a=6,2 \cdot 10^{-6} \mathrm{~m}^{2} / \mathrm{s}$; thermal conductivity - $\lambda=40 \mathrm{~W} /\left(\mathrm{m} \cdot{ }^{\circ} \mathrm{C}\right) ;$ heat-transfer coefficient; - $\alpha=455 \mathrm{~W} /\left(\mathrm{m}^{2} \cdot{ }^{\circ} \mathrm{C}\right)$; $T_{z d}^{*}=1220^{\circ} \mathrm{C}$; heating time $-\tau^{*}=32 \mathrm{~s}$. Dependency diagrams of power and temperature distribution are shown on figure 1,2 and figure 3, 4 correspondingly. 


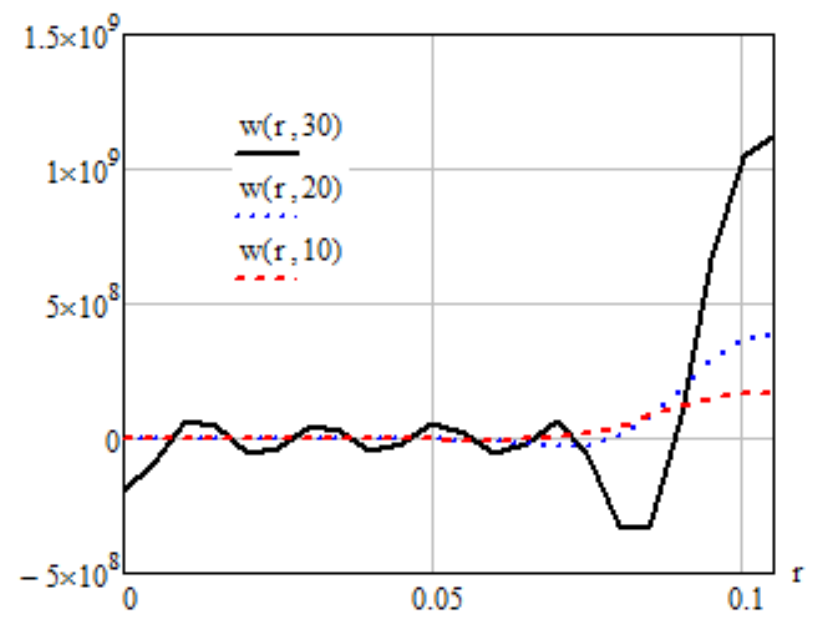

Figure 1. The distribution of optimal capacity of sources for different time points

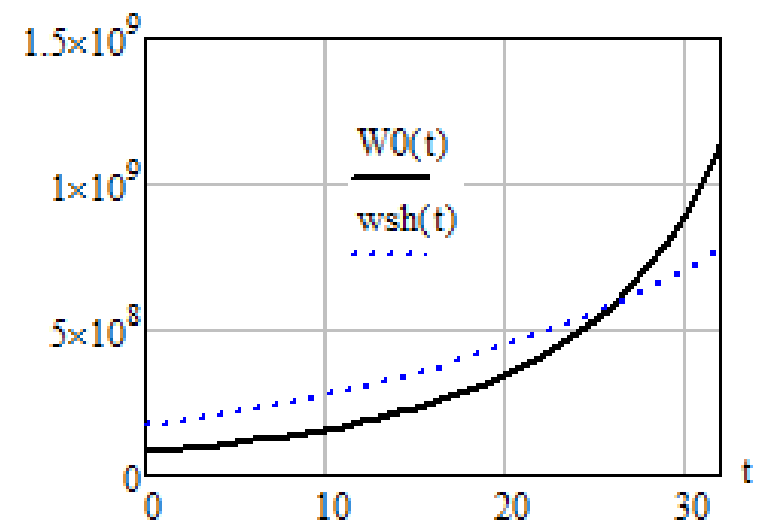

Figure 2. The change of specific capacity by optimal law (28) - W0 (t) and the law of physical work [8]-wsh (t)

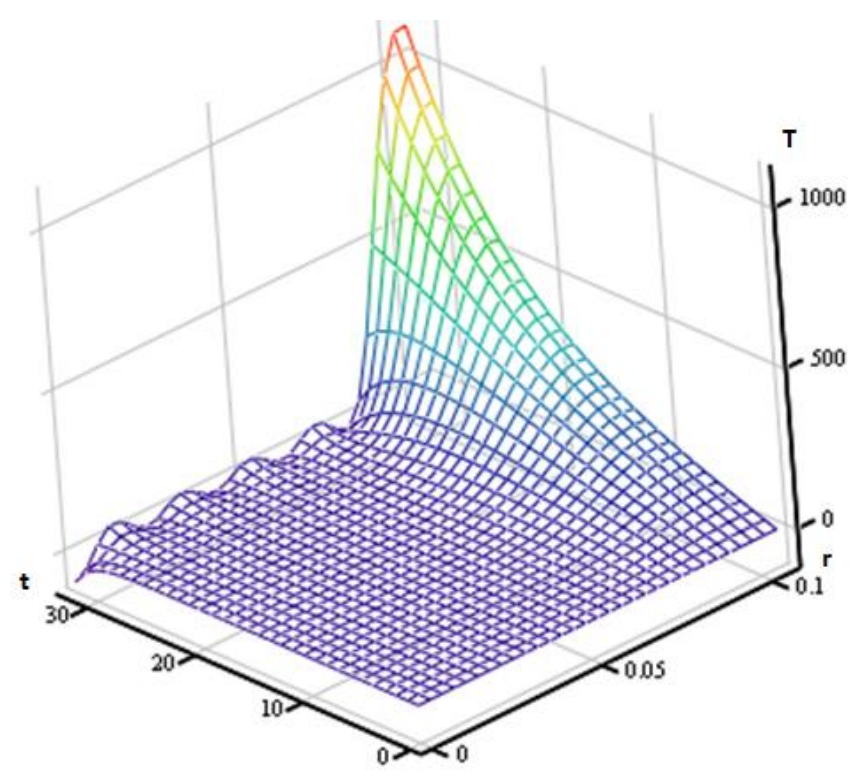

Figure 3. Temperature field of the plate 


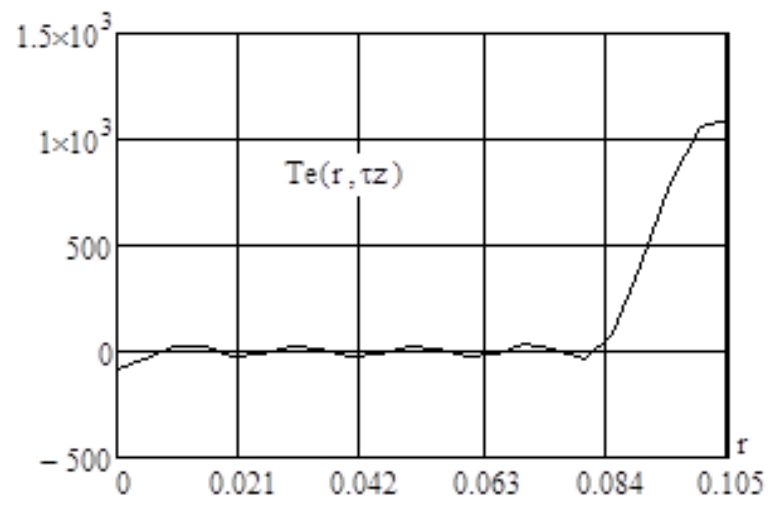

Figure 4. Temperature distribution within the plate at final time point

Conclusions. Energy conservation obtained as a result of application of optimal law for source capacity change (28) in comparison with heating at constant capacity.

$$
\Delta_{1}=\frac{W_{o p t}-W^{*}}{W^{*}} \cdot 100 \%=36,832 \%
$$

Energy conservation obtained as a result of application of optimal law for source capacity change (28) in comparison with heating by the law of physical work [8]

$$
\Delta_{2}=\frac{W_{o p t}-W_{s h}}{W_{s h}} \cdot 100 \%=15,775 \%
$$

\section{References}

1. Sluhockiy A. E. Induktory dlya idukcionnogo nagreva. Leningrad: Energiya, 1974. 264 p. [In Russian].

2. Podstryhach Ya. S., Shvets' R. N. Termoupruhost' tonkih obolochek. Kiev: Naukova dumka, 1978. 343 p. [In Russian].

3. Podstryhach Ya. S., Koliano Yu. M., Semerak M. M. Temperaturnye polia i napriagenia v elektrovakuumnyh priborah. Kiev: Naukova dumka, 1981. 342 p. [In Russian].

4. Shabliy O. M., Pulka Ch. V., Senchushyn V. S., Havryliuk V. Ya. Rozroblennya energooshchadnyh nahrivalnyh system dlya indukciynoho naplavlennya detalei sil's'kogospodarskyh mashyn. Visnyk TNTU. No. 4. 2011. P. 107-120. [In Ukrainian].

5. Shabliy O. M., Pulka Ch. V., Havryliuk V. Ya., Senchushyn V. S. Regymy indukciynogo naplavlennya detaley z urahuvannyam vykorystannya teplovyh I electromagnitnyh poliv. Visnyk TNTU. Vol. 73. No. 1. 2014. P. 109-118. [In Ukrainian].

6. Ryabcev I. A. Naplavka detalei mashyn i mehanizmov. Kiev: Ecotehnologia, 2004. 160 p. [In Russian].

7. Tkachev V. N. Iznos i povyshenie dolgovechnosti detaley selskohoziaestvennyh mashyn. Moskva: Mashynostroenie, 1971. 264 p. [In Russian].

8. Shabliy O. N., Pulka Ch. V., Budzan B. P. Optimizacyia energozatrat pri indukcyonnoy naplavke tonkostennyh diskov. Avtomaticheskaya svarka. Vol. 487. No. 1. 1987. P. 36-39. [In Russian].

9. Shabliy O. N., Pulka Ch. V., Budzan B. P. Puti ekonomii elektroenergii pri indukcyonnoy naplavke tonkostennyh diskov. Avtomaticheskaya svarka. Vol. 429. No. 12. 1988. P. 56-58. [In Russian].

\section{Список використаної літератури}

1. Слухоцки А. Е., Рыскин С. Е. Индукторы для индукционного нагрева. Л.: Энергия, 1974. 264 с.

2. Подстригач Я. С., Швец Р. Н. Термоупругость тонких оболочек. К.:Наукова думка, 1978. 343 с.

3. Подстригач Я. С., Коляно Ю. М., Семерак М. М. Температурные поля и напряжения в электровакуумных приборах. К.:Наукова думка, 1981.342 с. 
4. Шаблій О. М., Пулька Ч. В., Сенчишин В. С., Гаврилюк В. Я. Розроблення енергоощадних нагрівальних систем для індукційного наплавлення деталей сільськогосподарських машин. Вісник ТНТУ. 2011. № 4. С. 107-120.

5. Шаблій О. М., Пулька Ч. В., Гаврилюк В. Я., Сенчишин В. С. Режими індукційного наплавлення деталей із урахуванням використання екранування теплових і електромагнітних. Вісник ТНТУ. 2014. № 1 (73). С. 109-118.

6. Рябцев И. А. Наплавка деталей машин и механизмов. Киев: Экотехнология, 2004. 160 с.

7. Ткачев В.Н. Износ и повышение долговечности деталей сельскохозяйственных машин. М.: Машиностроение, 1971. 264 с.

8. Шаблий О. Н., Пулька Ч. В., Будзан Б. П. Оптимизация энергозатрат при индукционной наплавке тонкостенных дисков. Автоматическая сварка. 1987. № 1 (406). С. 36-39.

9. Шаблий О. Н., Пулька Ч. В., Будзан Б. П. Пути экономии электроэнергии при индукционной наплавке тонкостенных дисков. Автоматическая сварка. 1988. № 12 (429). С. 56-58.

\title{
УДК 621.793.927.7
}

\section{ОПТИМАЛЬНЕ КЕРУВАННЯ ІНДУКЦІЙНИМ НАГРІВОМ У ПРОЦЕСАХ НАПЛАВЛЕННЯ ТОНКИХ ФАСОННИХ ДИСКІВ}

\author{
Володимир Гаврилюк; Володимир Михайлишин; \\ Михайло Михайлишин; Чеслав Пулька
}

\author{
Тернопільський начіональний технічний університет імені Івана Пулюя, \\ Тернопіль, Україна
}

\begin{abstract}
Резюме. Індукиійний нагрів широко застосовується в процесах наплавлення, які використовуються для відновлення спрацьованих або для зміџнення робочої поверхні при виготовленні нових деталей, які в основному застосовуються в сільськогосподарському машинобудуванні. Перевагами індукційного нагрівання $\epsilon$ висока концентрація енергї в зоні нагріву, щзо дозволяє підвищити продуктивність процесу за рахунок скорочення часу. Однак індукиійний нагрів має ряд недоліків, а саме: електромагнітне випромінювання та надмірні витрати електроенергії, які впливають на здоров'я оточуючих $і$ на оплату послуг за використану енергію. 3 метою заощадження електроенергї, авторами розроблений більш точний оптимальний закон зміни в часі потужності теплових джерел. Числові розрахунки виконувалися для процесу індукційного наплавлення високочастотним генератором, тонких фасонних дисків шихтою - порошкоподібним твердим сплавом із застосуванням двовиткового кільцевого індуктора. Дані для яких виконувалися розрахунки: геометричні розміри - радіус диска 105 тm, радіус поверхні диска без иихти $92 \mathrm{~mm}$, иирина шару иихти $13 \mathrm{~mm}$, товщиназ mm, матеріал диска - сталь $\mathrm{Cm} 3 \mathrm{cn}$ із заданими теплофізичними характеристиками, температура нагрівання- $1220^{\circ} \mathrm{C}, 4 а \mathrm{c}$ нагрівання - 32 с. Проведені числові обчислення дозволяють вивести оптимальний закон зміни потужності джерел. При виконанні точніших розрахунків із врахуванням всіх необхідних коефіцієнтів приводить до економії електроенергії на $36,8 \%$ порівняно з нагрівом при постійній потужності в часі $i$ на 15,8\% - з нагрівом за оптимальним законом, але без точного урахування теплофізичних коефіцієнтів. Приведені графічні залежності дозволяють ознайомитися із розподілом та зміною оптимальної потужності джерел для різних моментів часу, а також показують зміну температурного поля в залежності від часу та радіусу наплавлюваного фасонного диска.
\end{abstract}

Ключові слова: індукиійний нагрів, наплавлення, оптимальний закон, потужність, температура, тонкий фасонний диск. 